\title{
The effects of liraglutide in mice with diet-induced obesity studied by metabolomics
}

\author{
Martina Bugáňová1,2, Helena Pelantová1, Martina Holubová3, Blanka Šedivá4, \\ Lenka Maletínská3 , Blanka Železná3, Jaroslav Kuneš3,5, Petr Kačer1,2, \\ Marek Kuzma' and Martin Haluzík6,7
}

${ }^{1}$ Institute of Microbiology, Academy of Sciences of the Czech Republic, Prague, Czech Republic 2Faculty of Chemical Technology, University of Chemistry and Technology Prague, Prague, Czech Republic Institute of Organic Chemistry and Biochemistry, Academy of Sciences of the Czech Republic, Prague, Czech Republic ${ }^{4}$ Faculty of Applied Sciences, University of West Bohemia, Plzeň, Czech Republic IInstitute of Physiology, Academy of Sciences of the Czech Republic, Prague, Czech Republic ${ }^{6}$ Centre for Experimental Medicine and Diabetes Centre, Institute for Clinical and Experimental Medicine, Prague, Czech Republic

${ }^{7}$ Institute of Medical Biochemistry and Laboratory Diagnostics, 1st Faculty of Medicine, Charles University and General University Hospital in Prague, Prague, Czech Republic

Correspondence should be addressed to M Haluzík Email mhalu@If1.cuni.cz

\begin{abstract}
Liraglutide is the glucagon-like peptide-1 receptor agonist widely used for the treatment of type 2 diabetes mellitus. Recently, it has been demonstrated to decrease cardiovascular morbidity and mortality in patients with type 2 diabetes and high cardiovascular risk. Although the major modes of liraglutide action are well-known, its detailed action at the metabolic level has not been studied. To this end, we explored the effect of 2-week liraglutide treatment in C57BL/6 male mice with obesity and diabetes induced by 13 weeks of high-fat diet using NMR spectroscopy to capture the changes in urine metabolic profile induced by the therapy. The liraglutide treatment decreased body and fat pads weight along with blood glucose and triglyceride levels. NMR spectroscopy identified 11 metabolites significantly affected by liraglutide treatment as compared to high-fat diet-fed control group. These metabolites included ones involved in nicotinamide adenine dinucleotide metabolism, $\beta$-oxidation of fatty acids and microbiome changes. Although majority of the metabolites changed after liraglutide treatment were similar as the ones previously identified after vildagliptin administration in a similar mouse model, the changes in creatinine, taurine and trigonelline were specific for liraglutide administration. The significance of these changes and its possible use in the personalization of antidiabetic therapy in humans requires further research.
\end{abstract}

Journal of Endocrinology (2017) 233, 93-104

\section{Introduction}

Type 2 diabetes mellitus (T2DM) represents one of the most important challenges of current medicine owing to its rapidly increasing prevalence and the accompanying risk of chronic complications that markedly contribute to increased morbidity and mortality of these patients (O'Rahilly 1997, Haffner 2003). During the last decade, http://joe.endocrinology-journals.org DOI: $10.1530 / J O E-16-0478$
() 2017 Society for Endocrinology Printed in Great Britain
Published by Bioscientifica Ltd 
multiple novel classes of antidiabetic therapies have been introduced to the clinical practice (Abdul-Ghani \& DeFronzo 2008, Drucker et al. 2010). Nevertheless, the exact algorithm of T2DM treatment, and in particular, the identification of patients' characteristics predicting optimal efficacy of particular therapy is still a matter of an intensive discussion and ongoing research (Davidson 2013). As T2DM has a very complicated etiopathogenesis with many important factors being involved, complex methods able to capture these multiple changes are required for proper identification of predictive markers of efficacy of different therapies.

NMR-based metabolomics in biofluids (e.g. urine or blood plasma) has become a strong tool for monitoring of different physiological and pathological processes as well as the response to the various therapeutic interventions (Nicholson et al. 1999). Its advantage lies in the fact that it reflects generally instantaneous changes within the organism. Metabolomics thus could potentially bring an innovative view on the efficacy and prediction of long-term benefits of well-established therapeutic agents including antidiabetic therapies.

Incretin-based therapies represent one of the most promising approaches in $\mathrm{T} 2 \mathrm{DM}$ treatment owing to good efficacy and the fact that they do not have major side effects such as hypoglycemia and weight gain (Drucker et al. 2011, Deacon et al. 2012). These therapies include either dipeptidylpeptidase-4 (DPP-4) inhibitors or glucagon-like peptide-1 receptor agonists (GLP1RAs) (Brunton 2014). DPP-4 inhibitors are presumably a suitable well-tolerated choice for either dual-therapy with metformin or for metformin substitution in case of its contraindication or intolerance (Inzucchi et al. 2015). GLP-1RAs are not only very efficacious in improving diabetes control but they also induce weight loss along with improved blood pressure and circulating lipid levels (Davidson 2011). They can be used in a wide range of patients with T2DM including those on insulin therapy. Furthermore, the most widely used representative of this class liraglutide has been recently shown to significantly decrease the risk of cardiovascular death and cardiovascular complications in high-risk patients with T2DM (Marso et al. 2016).

Despite many experimental and clinical data on the metabolic effects of liraglutide, some aspects of its mode of action still remain to be clarified in more detail. To this end, we performed our metabolomic study to shed more light on complex metabolic changes induced by liraglutide administration. The present study is primarily focused on the metabolomic analysis of the effects of subcutaneously administered liraglutide in a mouse model of diet-induced obesity (DIO) and T2DM. The changes in metabolic fingerprint of urine after 14-day treatment by liraglutide were explored using NMR spectroscopy. To the best of our knowledge, this is the first study combining metabolomics with a thorough phenotypic characterization of the effects of liraglutide in a mouse model of obesity and T2DM.

\section{Materials and methods}

\section{Animals, diets and treatment}

All experiments were conducted according to the ethical guidelines for animal experiments and the Czech Republic law No. 246/1992 and were approved by the Committee for Experiments with Laboratory Animals of the Academy of Sciences of the Czech Republic.

Three-week-old inbred C57BL/6 male mice were obtained from Charles River. The mice were housed under controlled conditions at a constant temperature of $22 \pm 2^{\circ} \mathrm{C}$, relative humidity $45-65 \%$ and fixed daylight cycle (06:00-18:00 h), with 5 mice per cage. The animals were provided free access to water and a standard rodent chow diet Ssniff R/M-H containing 33, 9 and 58\% of calories from protein, fat and carbohydrate, respectively (Ssniff Spezialdiäten GmbH, Soest, Germany).

From 8 weeks of age, C57BL/6 mice were fed a highfat (HF) diet to induce obesity. The energy content of the HF diet was $5.3 \mathrm{kcal} / \mathrm{g}$, with 13,60 and $27 \%$ of the calories derived from proteins, fats and carbohydrates, respectively. The diet was composed of $40 \%$ standard chow (Ssniff R/M-H), 34\% powdered cow-milk-based human baby formula, 25\% lard and 1\% corn starch (w/w) (Kopecký et al. 1996). Mice resistant to HF diet were withdrawn from the experiment. After 13 weeks of HF feeding, the average body weight of mice used for the experiments was $51.14 \pm 0.56 \mathrm{~g}$, which is approximately twice the weight of the age-matched lean mice.

At this point, the mice were divided into two groups with 10 animals per group and placed into separate cages with free access to the HF diet and water. Twoweek subcutaneous (s.c.) administration of liraglutide was initiated, while the mice were kept on the HF diet. Liraglutide was injected twice a day at a dose of $0.2 \mathrm{mg} / \mathrm{kg}$ in a dosing volume of $0.15 \mathrm{~mL}$. The control group received equal volume of saline. Food intake and body weight were monitored daily during the dosing period. 


\section{Biometric, biochemical and hormonal parameters}

Blood sampling for biochemical analyses and tissue dissection At the end of the experiment, the mice were killed by decapitation. The trunk blood was collected, and the plasma was separated and stored at $-20^{\circ} \mathrm{C}$. The subcutaneous adipose tissue (SCAT), visceral adipose tissue (VAT), brown adipose tissue (BAT), perirenal adipose tissue and liver were dissected and weighed. Total adipose tissue mass was calculated as $\mathrm{SCAT}+\mathrm{VAT}+$ perirenal adipose tissue.

Determination of hormonal and biochemical parameters Plasma insulin concentrations were measured using an RIA assay (Millipore). Plasma leptin concentrations were determined by ELISA (Millipore). Blood glucose levels were measured using a Glucocard glucometer (Arkray, Kyoto, Japan). Plasma triglyceride levels were measured using a quantitative enzymatic reaction (Sigma). All measurements were performed according to the manufacturer's instructions.

Statistical analysis Data obtained from biometric, biochemical and hormonal measurements are presented as the mean \pm S.E.M. Statistical analysis was performed by unpaired $t$-test or repeated measures ANOVA with Bonferroni post hoc test, as indicated in figures and tables legends. The differences between the control and treated group were considered significant at $P<0.05$.

\section{NMR-based metabolomics}

Sample collection Urine samples for NMR-based metabolomics were collected twice: after 13 weeks on HF diet (before the start of liraglutide treatment) and subsequently at the end of the experiment, after 2 weeks of treatment. Urine was collected overnight (from 17:00 h to $08: 00 \mathrm{~h}$ ), whereas mice were housed in individual metabolic cages (Tecniplast, Buguggiate, Italy) without access to food. To prevent bacterial contamination, $25 \mu \mathrm{L}$ $0.1 \%(\mathrm{w} / \mathrm{v}) \mathrm{NaN}_{3}$ was added to each sample of urine. Afterward, the samples were stored at $-80^{\circ} \mathrm{C}$ until the NMR analysis.

Sample preparation and NMR experiments Immediately before the NMR experiments, the urine samples were thawed gradually at room temperature. The samples were then centrifuged at $13,684 \boldsymbol{g}$ for $5 \mathrm{~min}$. A $200 \mu \mathrm{L}$ aliquot of supernatant was mixed with $340 \mu \mathrm{L}$ $\mathrm{H}_{2} \mathrm{O}$ and $60 \mu \mathrm{L}$ phosphate buffer $\left(1.5 \mathrm{M} \mathrm{KH}_{2} \mathrm{PO}_{4}\right.$ in $\mathrm{D}_{2} \mathrm{O}$ containing $2 \mathrm{mM} \mathrm{NaN}_{3}$ and $0.1 \%(\mathrm{w} / \mathrm{v})$ trimethylsilyl propionic acid (TSP), $\mathrm{pH} 7.4$ ) and placed in a 5-mm NMR tube. The NMR spectra were acquired on a $600 \mathrm{MHz}$ Bruker Avance III spectrometer (Bruker BioSpin, Rheinstetten, Germany) equipped with a 5-mm TCI cryogenic probe head. The experiments were performed at $300 \mathrm{~K}$. Tuning, matching, shimming and adjusting of the $90^{\circ}$ pulse length were carried out for each sample in automatic mode.

1D proton spectra were acquired using a Carr-PurcellMeiboom-Gill (CPMG) pulse program (Bruker pulse sequence cpmgpr1d) with presaturation during relaxation delay (4s) using a $25-\mathrm{Hz}$ saturation pulse centered on water resonance; number of scans $(\mathrm{NS})=64$; number of data points $(\mathrm{TD})=64 \mathrm{k}$; spectral width $(\mathrm{SW})=20 \mathrm{ppm}$; echo time $=0.3 \mathrm{~ms}$ and loop for T2 filter $=126$. The CPMG pulse sequence was applied to filter out the undesirable signals of proteins, which naturally occur in mouse urine and thus complicate the spectra evaluation (Pelantová et al. 2015). To facilitate metabolite identification, a short J-resolved experiment with presaturation (Bruker pulse sequence jresgpprqf, $\mathrm{NS}=2, \mathrm{SW}=16 \mathrm{ppm}, \mathrm{TD}=8 \mathrm{k}$, number of increments $=40, \mathrm{SW}=78.125 \mathrm{~Hz}$ in the indirect dimension and relaxation delay $=2 \mathrm{~s}$ ) was executed for each sample. The structural information obtained by $1 \mathrm{D}$ experiments was supported and validated by the $2 \mathrm{D}$ experiments. ${ }^{1} \mathrm{H}-{ }^{1} \mathrm{H}$ correlation spectroscopy (COSY) and ${ }^{1} \mathrm{H}-{ }^{13} \mathrm{C}$ heteronuclear multiple-quantum correlation (HMQC) experiments (Bruker pulse sequences cosygpprqf and hmqcphpr) were applied on representative samples of urine.

Data preprocessing Acquired spectral data were processed using Topspin 3.2 software (Bruker BioSpin). Free induction decays (FIDs) were multiplied by an exponential window function $(\mathrm{LB}=0.3 \mathrm{~Hz})$ before the Fourier transformation. The spectra were automatically phased, baseline corrected and referenced to TSP (0.00 ppm). The processed CPMG spectra (0.10-10.0 ppm) were uniformly binned to 0.01-ppm intervals in Amix 3.9.14 software (Bruker Biospin). The regions containing residual water resonance $(4.60-4.90 \mathrm{ppm})$ and urea resonance $(5.60-6.00 \mathrm{ppm})$ were excluded from the spectra prior to statistical analysis.

\section{Statistical analysis of NMR data}

Multivariate statistical analysis To explore potential metabolic changes induced by liraglutide therapy, the binned data extracted from proton spectra were submitted to MetaboAnalyst 3.0 software (Xia et al. 2009, 2015) and

Published by Bioscientifica Ltd. 
Matlab software (MATLAB version 8.6; The MathWorks Inc., Natick, MA, USA) for statistical evaluation. Spectra were normalized to the total spectral area to compensate various dilutions of individual urine samples. Meancentered and Pareto-scaled data were subjected to principal component analysis (PCA) to visualize the grouping and to detect outliers. Afterward, the partial least squarediscriminant analysis (PLS-DA) was executed to identify bins contributing to the separation of the treated and the control group of mice. The PLS-DA characterized each bin with variable importance in projection (VIP) scores based on its contribution to the differentiation of the treated and control mice. The bins with VIP $>1.5$ were considered as important in our model. The leaveone-out validation was used to determine the quality of the model and to choose the correct number of latent variables with respect to the performance of the model on the validation data set. The fractions of explained variation $\left(R^{2}\right)$ and predicted variation $\left(Q^{2}\right)$ were examined to provide more detailed evaluation of the trained model. Model validation was completed using the permutation test (2000 permutations).

Univariate statistical analysis The binned spectra were in parallel examined using univariate statistics. As the data within investigated groups were suspected to possess unequal variation and non-normal distribution, both the unpaired $t$-test and nonparametric WilcoxonMann-Whitney test were performed. The changes of bin intensities with $P<0.05$ resulted from at least one test were considered as important for further discussion.

Statistically significant bins resulted from both above-mentioned multivariate and univariate statistical approaches were assigned using Chenomx NMR Suite 7.6 (Chenomx Inc., Edmonton, AB, Canada), the Human Metabolome Database (HMDB) and previously published metabolomic papers (Boulange et al. 2013).

Metabolite quantitation To exclude the possibility that some significant metabolic changes were not captured through untargeted evaluation of individual bins, we subsequently applied metabolomic profiling approach also. Using the method described in the previous paragraph, we identified as many metabolites present in spectra as possible. Each identified metabolite was quantified using one representative signal whose intensity was calculated by summing up the intensities of respective adjacent $0.01 \mathrm{ppm}$ bins to cover the whole signal width. For selection of an appropriate statistical method (parametric or nonparametric), we performed the Lilliefors test of normality. The resulting metabolites intensities were then re-examined using unpaired $t$-test or nonparametric Wilcoxon-Mann-Whitney test, and the data were visualized in box-plots. To eliminate the problem with multiple testing, we used BenjaminiHochberg-Yekutieli procedure (Benjamini \& Yekutieli 2001) for controlling the false discovery rate and calculating the corrected $P$ value. Depending on results of normality tests, the Pearson's correlation or Spearman's correlation was used to determine the potential relations between metabolites intensities and standard biochemical or biometric parameters, all measured at the end of the experiment.

\section{Results}

\section{Effect of liraglutide on biometric, biochemical and hormonal parameters in DIO mice}

The 2-week administration of liraglutide to DIO mice tended to decrease food intake (Fig. 1A) and significantly decreased body weight (Fig. 1B) and the weights of SCAT, VAT, perirenal and total adipose tissue as compared with the control group (Table 1). The values are presented as relative to body weight to eliminate the direct association of organs mass reduction to weight loss. The treatment with liraglutide also significantly decreased fasting glucose, leptin and triglyceride levels, and it also tended to reduce blood insulin levels (Table 1).

One mouse from liraglutide-treated group had not survived till the end of the experiment; therefore, this group consists of only 9 animals.

\section{Results of NMR-based metabolomics}

Untargeted analysis Visualization of the data through PCA did not detect any outliers (data not shown). The PLS-DA with its untargeted approach was executed to unveil the spectral regions, which are responsible for the differences between treated group and control group. However, rather poor model validation (number of components: 3 ; accuracy: $0.79 ; R^{2}=0.89$; $Q^{2}=0.42 ; \quad P$ value of permutation test $(2000$ repetitions) $=0.19$ ) may indicate the potential risk of overfitting. Therefore, the changes captured by PLS-DA were used only as an additional information to those found using univariate tests.

All bins were also submitted to both parametric and nonparametric tests. The results obtained from both tests were overlapped and allowed us to determine the

Published by Bioscientifica Ltd. 
A

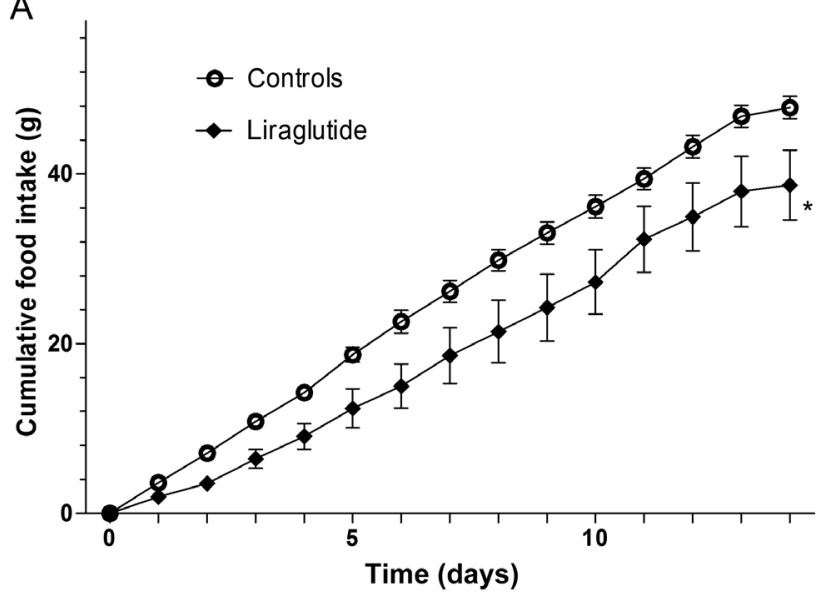

B

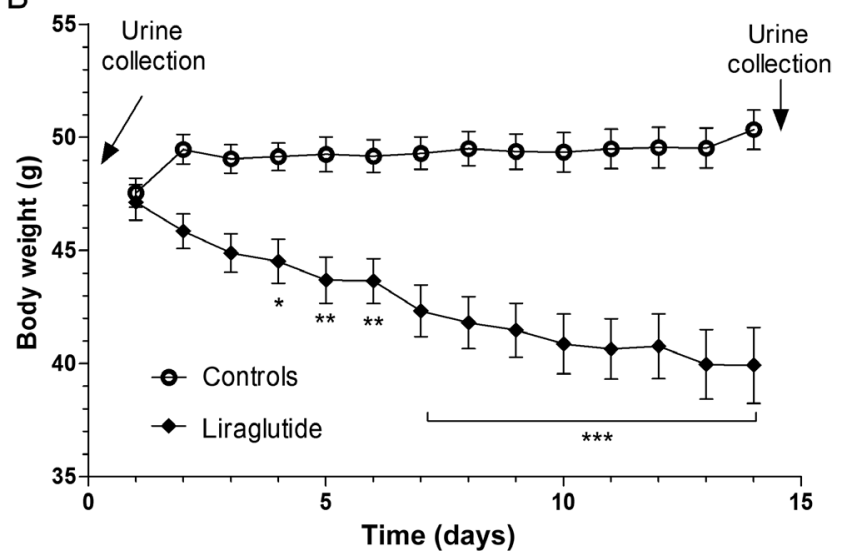

Figure 1

(A) The cumulative food intake and (B) the body weight of the DIO mice during the treatment with liraglutide. The data are presented as the mean \pm S.E.M. Statistical analysis was performed using the repeated measures ANOVA followed by the Bonferroni post hoc test.

${ }^{*} P<0.05, * * P<0.01, * * * P<0.001$ vs control group.

significance of the changes all over the spectra. Table 2 lists metabolites with bins revealed as significant using PLS-DA and univariate tests. Table 2 shows that even though the PLS-DA failed to build a proper statistical model, there is still a large overlap of bins identified using univariate tests and PLS-DA.

Metabolomic profiling In the current study, 39 metabolites were identified using either Chenomx or HMDB database or previously published metabolomic studies. Figure 2 depicts the representative ${ }^{1} \mathrm{H}$ NMR spectrum of mouse urine with marked signals of identified metabolites. Table 3 shows the list of all identified metabolites with the corresponding signals used for identification. Based on the Lilliefors test of normality (data not shown), the unpaired $t$-test was applied on all
Table 1 Biometric, biochemical and hormonal parameters (either as absolute values or as relative values; organ weight/ body weight ratio) in mice treated with liraglutide vs control mice on high-fat diet at the end of the experiment.

\begin{tabular}{|c|c|c|}
\hline & Controls & Liraglutide \\
\hline $\begin{array}{l}\text { BAT/BW } \\
\text { SCAT/BW }\end{array}$ & $\begin{array}{l}0.52 \pm 0.02 \\
7.71 \pm 0.23\end{array}$ & $\begin{array}{l}0.60 \pm 0.04 \\
5.87 \pm 0.63\end{array}$ \\
\hline VAT/BW & $3.89 \pm 0.34$ & $2.75 \pm 0.31$ * \\
\hline Per/BW & $3.07 \pm 0.10$ & $1.82 \pm 0.29$ * \\
\hline Tot/BW & $14.66 \pm 0.37$ & $9.79 \pm 1.10 *$ \\
\hline Liver/BW & $3.61 \pm 0.21$ & $3.16 \pm 0.08$ \\
\hline Glucose (mmol/L) & $8.42 \pm 0.35$ & $4.74 \pm 0.49 *$ \\
\hline Insulin (ng/mL) & $3.18 \pm 0.31$ & $2.06 \pm 0.62$ \\
\hline Triglycerides (mg/mL) & $0.58 \pm 0.05$ & $0.42 \pm 0.05$ * \\
\hline Leptin (ng/mL) & $46.96 \pm 3.64$ & $18.00 \pm 4.65^{*}$ \\
\hline
\end{tabular}

The data are presented as the mean \pm S.E.M. Statistical analysis was performed using the unpaired $t$-test.

${ }^{*} P<0.05$ vs the control group.

AT, adipose tissue; BAT, brown AT; BW, body weight; Per, perirenal AT; SCAT, subcutaneous AT; Tot, total AT; VAT, visceral AT.

quantified signals (except overlapped signals or those of poor intensity). It resulted in eleven metabolites significantly affected by the treatment (Fig. 3).

In the region between 0.8 and $2.40 \mathrm{ppm}$, the statistical analyses revealed several resonances (around 0.85, $1.30,1.60$ and $2.30 \mathrm{ppm}$ ) where intensities significantly increased as a result of liraglutide treatment. They resulted from an overlap of signals belonging to the various short-chain (4 or 5 carbon atoms long) carboxylic acid (SCCA) derivatives. Unfortunately, due to their common structural features, we were not able to clearly identify particular compounds (except hexanoylglycine). Therefore, these changes will not be further discussed.

Two-week treatment with liraglutide significantly decreased urine levels of nicotinamide metabolites 2-PY (N1-methyl-2-pyridone-5-carboxamide), 4-PY (N1-methyl-4-pyridone-3-carboxamide) and trigonelline compared with the control group on HF diet.

On the contrary, the concentrations of glycine derivatives dimethylglycine and phenylacetylglycine were significantly increased along with the levels of both creatinine and creatine. Liraglutide treatment also increased the levels of 3-indoxyl sulfate while reducing concentrations of cis-aconitate and taurine. The levels of hexanoylglycine and SCCAs derivatives were significantly increased by the therapy compared with HF diet control group.

Based on the results of Lilliefors test of normality, significantly changed metabolites were correlated with significantly influenced biochemical or biometric parameters (their relative values, i.e. values related to the

Published by Bioscientifica Ltd 

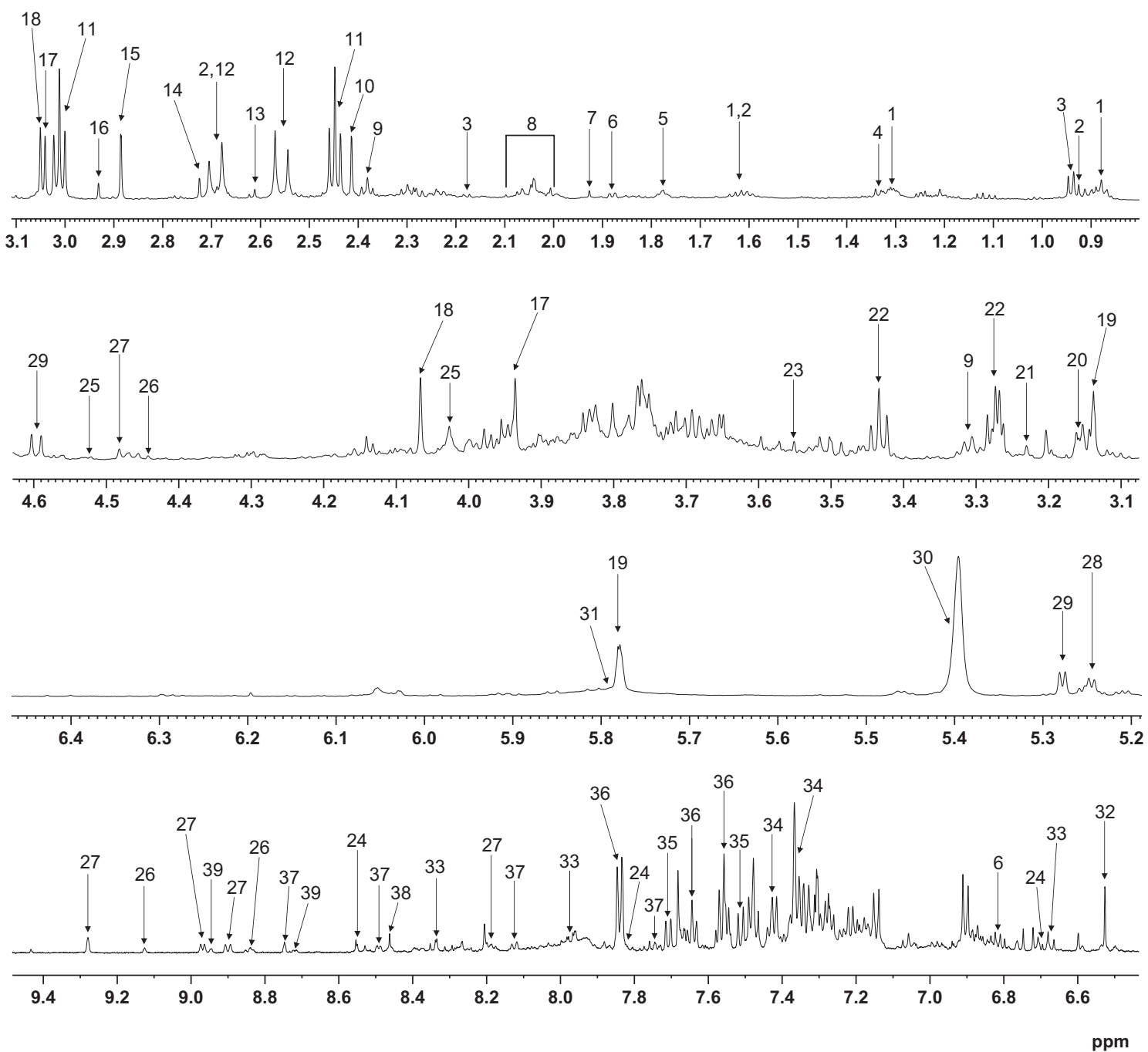

Figure 2

Representative ${ }^{1} \mathrm{H}$ NMR spectrum of urine from liraglutide-treated mice. Metabolite assignments for the numbers are given in Table 3.

body weight) using Pearson's correlation. The correlation revealed several statistically significant relationships that are depicted in Table 4 . All three nicotinamide metabolites 2-PY, 4-PY and trigonelline as well as cis-aconitate were strongly or moderately positively correlated with all investigated parameters (body weight, glucose, leptin, and the relative mass of total and perirenal fat and SCAT) only correlation between cis-aconitate and the relative mass of SCAT was not observed. Taurine strongly positively correlated with glucose, leptin and relative mass of total fat, and also moderately correlated with body weight and relative weight of perirenal fat.

In case of 3-indoxyl sulfate, a moderate inverse correlation was found with body weight, leptin, glucose and the relative mass of perirenal and total fat. The urine levels of dimethylglycine strongly inversely correlated with body weight, glucose and leptin as well as the relative mass of SCAT, perirenal and total fat. Phenylacetylglycine moderately inversely correlated with only glucose and relative mass of total fat. Hexanoylglycine and undifferentiated SCCAs derivatives correlated inversely with body weight, glucose and leptin. Finally, creatine inversely correlated with glucose and the relative mass of SCAT.

\section{Discussion}

Incretin-based therapies are among the most widely used treatment options in patients with T2DM owing to their good efficacy and safety. Here, we used NMR-based metabolomics to explore complex metabolic effects of GLP-1 RA liraglutide and its relationship with biometric, 
Table 2 The changes in metabolites identified by PLS-DA and univariate tests in urine of DIO mice after 2-week treatment with liraglutide.

\begin{tabular}{|c|c|c|}
\hline & PLS-DA & Univariate tests \\
\hline \multicolumn{3}{|l|}{ Decrease } \\
\hline 2-Oxoglutarate & $\downarrow$ & - \\
\hline 2-PY & - & $\downarrow$ \\
\hline 4-PY & - & $\downarrow$ \\
\hline Citrate & $\downarrow$ & - \\
\hline Trigonelline & - & $\downarrow$ \\
\hline cis-Aconitate & $\downarrow$ & $\downarrow$ \\
\hline Taurine & $\downarrow$ & $\downarrow$ \\
\hline \multicolumn{3}{|l|}{ Increase } \\
\hline 3-Indoxyl sulfate & - & $\uparrow$ \\
\hline Allantoin & $\uparrow$ & - \\
\hline N-carbamoyl- $\beta$-alanine & $\uparrow$ & - \\
\hline Hexanoylglycine + SCCAs derivatives & $\uparrow$ & $\uparrow$ \\
\hline Creatine & $\uparrow$ & $\uparrow$ \\
\hline Creatinine & $\uparrow$ & $\uparrow$ \\
\hline Dimethylglycine & $\uparrow$ & $\uparrow$ \\
\hline Phenylacetylglycine & $\uparrow$ & $\uparrow$ \\
\hline Vinylaceylglycine & $\uparrow$ & - \\
\hline
\end{tabular}

$\uparrow$ significant increase in concentration; $\downarrow$ significant decrease in concentration; - no significant change in metabolite intensity; significance of PLS-DA: VIP $>1.5$, significance of univariate tests $* P<0.05$. 2-PY, N1-methyl-2-pyridone-5-carboxamide; 4-PY, N1-methyl-4-pyridone-

3-carboxamide; SCCAs, short-chain carboxylic acids.

biochemical and hormonal changes induced by 2 weeks of liraglutide treatment. Such approach may help to further understand the exact mechanism of liraglutide action in man including its recently demonstrated protective effect against cardiovascular morbidity and mortality. To shed more light on the differences between the effects of GLP-1 RA and DPP-4 inhibitors on the metabolic level, we also discuss the differences in the metabolic fingerprint of 2-week liraglutide therapy with our previously published data exploring this approach in mice treated with DPP-4 inhibitor vildagliptin (Pelantová et al. 2016a).

As expected, liraglutide significantly decreased body weight due to reduction of body fat as described in numerous studies previously (van Can et al. 2014). It also significantly decreased the absolute liver weight (data not shown), which is in accordance with similar effects described in both experimental studies and in patients with T2DM (Jendle et al. 2009). By contrast, vildagliptin did not reduce the body weight in our previous study, underscoring the differences between more pronounced effects of GLP-1 RA on biometric parameters as compared to DPP-4 inhibitors, which is in agreement with previously published experimental and clinical data.
Table $3{ }^{1} \mathrm{H}$ NMR chemical shifts used for identification of metabolites in urine of DIO mice.

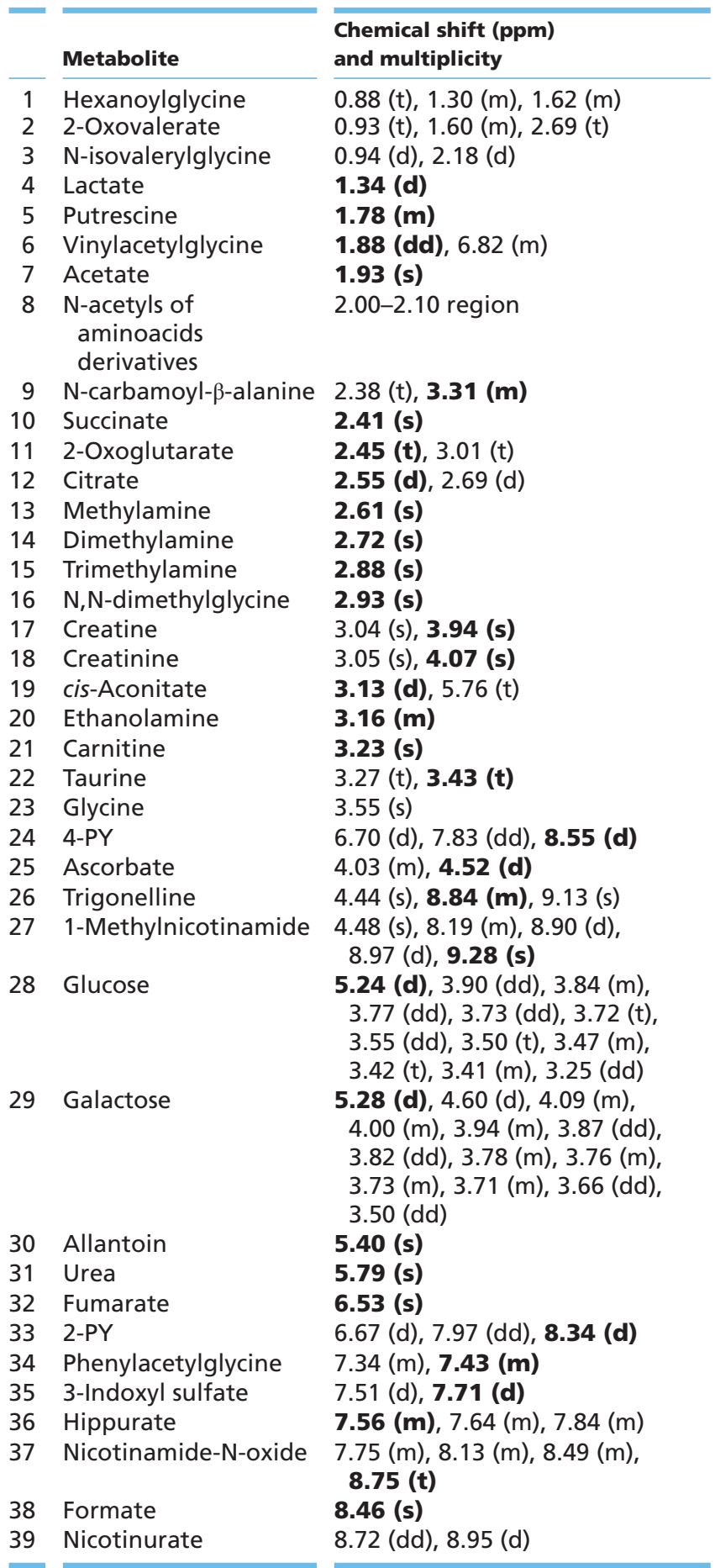

The table listed all unambiguously identified signals of particular metabolites. The signals used for quantification are in bold.

Signal multiplicity: (s)-singlet, (d)-doublet, (t)-triplet, (dd)-doublet of doublets, (m)-multiplet.

2-PY, N1-methyl-2-pyridone-5-carboxamide; 4-PY, N1-methyl-4-pyridone3-carboxamide.

Published by Bioscientifica Ltd 

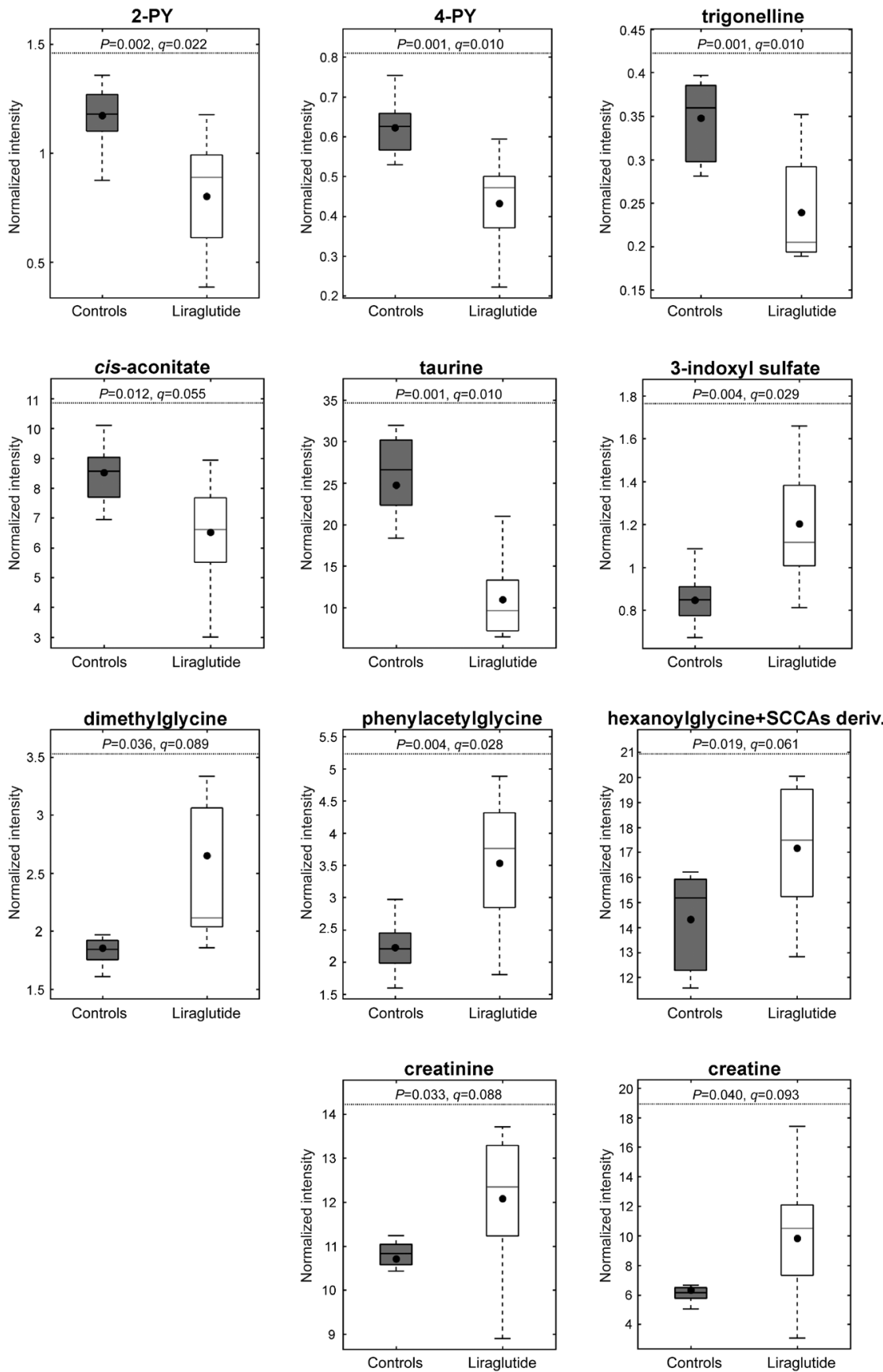

Figure 3

The box-plots with whiskers reflecting metabolites levels after the 2-week treatment with liraglutide in DIO mice. The results were calculated using unequal variance unpaired $t$-test followed by multiplicity adjustment using false discovery rate (FDR) (Benjamini \& Yekutieli 2001). Significance: $p$ ( $t$-test), q (FDR); liraglutide-treated vs control group. 2-PY, N1-methyl-2-pyridone-5carboxamide; 4-PY, N1-methyl-4-pyridone-3carboxamide; SCCAs, short-chain carboxylic acids; liraglutide-treated group is shown in white, and control group in grey.
NMR metabolic profiling of urine revealed a set of metabolites significantly affected by the 2 -week liraglutide therapy. Liraglutide treatment markedly decreased the levels of 2-PY (N1-methyl-2-pyridone-5-carboxamide) and 4-PY (N1-methyl-4-pyridone-3-carboxamide). These changes positively correlated with body weight, glucose and leptin levels and relative mass of SCAT, perirenal and total fat weights at the end of experiment. In our previous study with DPP-4 inhibitor vildagliptin, the same duration of treatment also decreased both these metabolites (Pelantová et al. 2016a) suggesting that their change could be related to the common mechanism of action of GLP-1 RA and DPP-4 inhibitors. The elevated levels of 2-PY and 4-PY (the end products of nicotinamide adenine dinucleotide metabolism) have previously been reported in urine of DIO mice (Boulange et al. 2013) and genetically obese $d b / d b$ mice (Salek et al. 2007). Moreover, the study by Salek and coworkers suggested that the nicotinamide metabolites, particularly 2 -PY might serve as potential biomarker of T2DM progression. Thus, an 
Table 4 Significant correlations between metabolites and biochemical or biometric parameters (either as absolute values or as relative values; organ weight/body weight ratio), all significantly affected by liraglutide treatment in DIO mice.

\begin{tabular}{|c|c|c|c|c|c|c|}
\hline & BW & Glucose & Leptin & Tot/BW & Per/BW & SCAT/BW \\
\hline 2-PY & $0.91 * * *$ & $0.81 * * *$ & $0.86 * * *$ & $0.85 * * *$ & $0.89 * * *$ & $0.78 * * *$ \\
\hline 4-PY & $0.86 * * *$ & $0.73 * * *$ & $0.90 * * *$ & $0.82 * * *$ & $0.85 * * *$ & $0.72 * * *$ \\
\hline Trigonelline & $0.62 * *$ & $0.67 * *$ & $0.61 * *$ & $0.58 * *$ & $0.53 *$ & $0.51 *$ \\
\hline cis-Aconitate & $0.75 * * *$ & $0.69 *$ & $0.60 * *$ & $0.62 * *$ & $0.71 * * *$ & - \\
\hline Taurine & $0.50 *$ & $0.65 * *$ & $0.67 * *$ & $0.62 * *$ & $0.49 *$ & - \\
\hline 3-Indoxylsulfate & $-0.47 *$ & $-0.52 *$ & $-0.53 *$ & $-0.58 * *$ & $-0.50 *$ & - \\
\hline Dimethylglycine & $-0.80 * * *$ & $-0.73 * * *$ & $-0.74 * \star \star$ & $-0.68 * *$ & $-0.79 * * *$ & $-0.61 * *$ \\
\hline Phenylacetylglycine & - & $-0.57 *$ & - & $-0.46 *$ & - & - \\
\hline Hexanoylglycine + SCCAs derivatives & $0.51 *$ & $-0.56 *$ & $-0.46 *$ & - & - & - \\
\hline Creatine & - & $-0.51 *$ & - & - & - & $-0.48 *$ \\
\hline
\end{tabular}

The table shows the results of Pearson's correlation expressed as correlation coefficients with respective significance: $* P<0.05, * * P<0.01$, $* * * P<0.001,-$ no significant correlation.

2-PY, N1-methyl-2-pyridone-5-carboxamide; 4-PY, N1-methyl-4-pyridone-3-carboxamide; BW, body weight; Per, perirenal adipose tissue; SCAT, subcutaneous adipose tissue; SCCAs, short-chain carboxylic acids; Tot, total fat.

alternative explanation of decreased 2-PY and 4-PY levels in our study may also lie in the improvement of diabetes compensation per se.

The reduced levels of trigonelline, which also belongs to the family of nicotinamide metabolites were strongly correlated with body weight, glucose and leptin levels measured after liraglutide treatment. Interestingly, vildagliptin treatment in our previous study did not have any effect on trigonelline levels (Pelantová et al. 2016a) suggesting that its changes might be specific for the treatment with liraglutide. Previous studies have suggested that changes in nicotinamide metabolism such as perturbations in trigonelline levels are present in T2DM and obesity although some of the data are rather inconsistent. For example, Jung and coworkers reported significantly higher levels of trigonelline in mice fed HF diet compared to those in mice fed high-carbohydrate diet (Jung et al. 2012). In contrast, the urinary trigonelline was decreased in the mice with MSG-induced obesity (Pelantová et al. 2016b). Our data suggest a possible role of altered nicotinamide metabolism in T2DM and its modification by liraglutide treatment.

3-Indoxyl sulfate is a mammalian-microbial urinary co-metabolite, which has been described to be altered as a consequence of changes in microbiome in rats (Swann et al. 2011, Liu et al. 2013). Moreover, 3-indoxyl sulfate may play a role in the regulation of the transcription of genes for cytochromes in liver (Schroeder et al. 2010), which can in turn influence the metabolic activity of xenobiotics. Thus, the increased levels of 3-indoxyl sulfate after liraglutide treatment may reflect a strengthened response to liraglutide administration. We detected moderate inverse correlations between 3-indoxyl sulfate and body weight, glucose, leptin levels, relative weight of perirenal and total fat at the end of the experiment suggesting possible association of this metabolite with T2DM and obesity. The same increase in 3-indoxyl sulfate concentration was found also in vildagliptin-treated mice in our previous study (Pelantová et al. 2016a). Increased 3-indoxyl sulfate concentrations in the urine in our study thus could also suggest the incretin-based therapies induced changes in intestinal microbiome. Alterations in bacterial microflora have been previously associated with obesity and T2DM, and its changes after various interventions have been reported (Hartstra et al. 2015). Unfortunately, as the changes in gut microflora were not assessed in our study, our data do not allow us to dissect its possible role in the changes in urine 3-indoxyl sulfate concentrations.

The levels of dimethylglycine (DMG) increased significantly in a response to liraglutide treatment. The only known pathway for DMG breakdown in mammals is through dehydrogenation of DMG. It was found that genetic variation in DMG dehydrogenase resulting in lower plasma DMG levels was related to higher blood glucose and increased insulin resistance (Magnusson et al. 2015). This relationship is in agreement with our current data, where urine DMG concentration after liraglutide treatment was strongly inversely related not only to blood glucose but also to body weight, serum leptin levels and relative weights of subcutaneous, perirenal and total adipose tissue. Similar to liraglutide, we detected increased DMG levels also after 2-week treatment with vildagliptin in our previous study (Pelantová et al. 2016a).

Phenylacetylglycine is another metabolite whose levels in urine were found to be associated with intestinal microbiome (Waldram et al. 2009). In our study, the concentrations of phenylacetylglycine significantly

Published by Bioscientifica Ltd 
increased after liraglutide treatment. Similar increase was seen in our previous study with vildagliptin (Pelantová et al. 2016a). The increased levels of creatine and creatinine could also be associated with altered metabolic activity of microorganisms, which produce specific enzymes for degradation of creatine and creatinine (Wyss \& Kaddurah-Daouk 2000). Although liraglutide increased both creatine and creatinine, vildagliptin did so only in case of creatine levels (Pelantová et al. 2016a). Clinical studies did not document any alterations in renal function with incretin-based therapies; therefore, it is unlikely that the changes of creatine and creatinine levels observed herein are related to changes in renal function.

Tricarboxylic acid (TCA) cycle has been previously reported to be upregulated by HF diet (Boulange et al. 2013). Therefore, we assumed TCA cycle could be altered also as a consequence of antidiabetic treatment in DIO mice. Administration of liraglutide decreased the level of cis-aconitate. The same decrease was found after treatment by vildagliptin (Pelantová et al. 2016a) in our previous study. Furthermore, vildagliptin reduced fumarate levels also, whereas no significant changes were seen with liraglutide treatment in the current study (Pelantová et al. 2016a). Collectively, all of the above-mentioned alterations may indicate the downregulation of TCA cycle induced by both therapies, which is in agreement with reported upregulation of TCA cycle in HF diet-fed mice (Boulange et al. 2013).

The treatment with liraglutide significantly decreased taurine levels compared with the untreated HF diet-fed group. Waterfield and coworkers suggested that increased urinary taurine could be a marker of liver damage (Waterfield et al. 1990, 1991). Their results demonstrated that the liver damage, in their case induced by toxic insult, increased taurine synthesis and consequently taurine excretion into urine also. Obesity and insulin resistance are commonly associated with liver steatosis (Fabbrini et al. 2010); therefore, the significant decrease in urine taurine after liraglutide treatment could indicate an improvement of liver steatosis through inhibition of taurine synthesis. Interestingly, 2-week treatment with vildagliptin in our previous study did not change taurine levels. It took 7 weeks of vildagliptin treatment to significantly decrease urine taurine concentrations (Pelantová et al. 2016a). These differences could be explained by more pronounced and direct effect of liraglutide on body weight and energy metabolism as compared to vildagliptin.

Hexanoylglycine originates from conjugation of glycine and hexanoyl-CoA, which is an intermediate metabolite of the $\beta$-oxidation. Thus, liraglutide-induced increase in hexanoylglycine might be a consequence of upregulated $\beta$-oxidation. The hypothesis of enhanced $\beta$-oxidation was discussed in our previous metabolomic study also (Pelantová et al. 2016a) where 2-week therapy with vildagliptin increased not only hexanoylglycine but also vinylacetylglycine. Boulange and coworkers found increased levels of hexanoylglycine after feeding with HF diet compared with mice fed a low-fat diet (Boulange et al. 2013). We suppose, that the inconsistency between our results and the findings of Boulange and coworkers could be explained by variations in the applied experimental protocol, e.g. the composition of HF diet administered to mice or urine collection method. However, the problem is rather comprehensive as the highest level of hexanoylglycine in Boulange study was observed in mice non-responsive to HF diet.

In summary, our study has revealed numerous metabolic effects induced by liraglutide treatment in mice with diet-induced obesity and type 2 diabetes mellitus. Majority of these changes were similar to changes induced by vildagliptin therapy as observed in our previous study. The significance of these changes and its possible use in the personalization of antidiabetic therapy in humans require further research.

\section{Declaration of interest}

The authors declare that there is no conflict of interest that could be perceived as prejudicing the impartiality of the research reported.

\section{Funding}

This research was financially supported by the Grant Agency of the Czech Republic (Grant No. GA13-14105S) and by institutional supports (RVO: 61388963, 67985823, 61388971, VFN64165 and MH CZ - DRO (Institute for Clinical and Experimental Medicine - IKEM, IN 000023001). This project was conducted within the 'Prague Infrastructure for Structure Biology and Metabolomics' with financial support of the Operational Program Prague - Competitiveness (Project No. CZ.2.16/3.1.00/24023). The authors acknowledge the project LO1509 and specific university research No 20-SVV/2016 of the Ministry of Education, Youth and Sports of the Czech Republic.

\section{Acknowledgements}

The authors would like to thank $\mathrm{H}$ Vysušilová for the excellent technical assistance.

\section{References}

Abdul-Ghani MA \& DeFronzo RA 2008 Inhibition of renal glucose reabsorption: a novel strategy for achieving glucose control in type 2 diabetes mellitus. Endocrine Practice 14 782-790. (doi:10.4158/EP.14.6.782)

Published by Bioscientifica Ltd. 
Benjamini Y \& Yekutieli D 2001 The control of the false discovery rate in multiple testing under dependency on JSTOR. Annals of Statistics 29 1165-1188. (doi:10.1214/aos/1013699998)

Boulange CL, Claus SP, Chou CJ, Collino S, Montoliu I, Kochhar S, Holmes E, Rezzi S, Nicholson JK, Dumas ME, et al. 2013 Early metabolic adaptation in C57BL/6 mice resistant to high fat diet induced weight gain involves an activation of mitochondrial oxidative pathways. Journal of Proteome Research 12 1956-1968. (doi:10.1021/pr400051s)

Brunton S 2014 GLP-1 receptor agonists vs. DPP-4 inhibitors for type 2 diabetes: is one approach more successful or preferable than the other? International Journal of Clinical Practice 68 557-567. (doi:10.1111/ijcp.12361)

Davidson MH 2011 Cardiovascular effects of glucagonlike peptide-1 agonists. American Journal of Cardiology 108 33B-41B. (doi:10.1016/j. amjcard.2011.03.046)

Davidson J 2013 The placement of DPP-4 inhibitors in clinical practice recommendations for the treatment of type 2 diabetes. Endocrine Practice 19 1050-1061. (doi:10.4158/EP12303.RA)

Deacon CF, Mannucci E \& Ahrén B 2012 Glycaemic efficacy of glucagonlike peptide- 1 receptor agonists and dipeptidyl peptidase- 4 inhibitors as add-on therapy to metformin in subjects with type 2 diabetes-a review and meta analysis. Diabetes, Obesity and Metabolism 14 762-767. (doi:10.1111/j.1463-1326.2012.01603.x)

Drucker DJ, Sherman SI, Gorelick FS, Bergenstal RM, Sherwin RS \& Buse JB 2010 Incretin-based therapies for the treatment of type 2 diabetes: evaluation of the risks and benefits. Diabetes Care $\mathbf{3 3}$ 428-433. (doi:10.2337/dc09-1499)

Drucker DJ, Sherman SI, Bergenstal RM \& Buse JB 2011 The safety of incretin-based therapies-review of the scientific evidence. Journal of Clinical Endocrinology and Metabolism 96 2027-2031. (doi:10.1210/ jc.2011-0599)

Fabbrini E, Sullivan S \& Klein S 2010 Obesity and nonalcoholic fatty live disease: biochemical, metabolic, and clinical implications. Hepatology 51 679-689. (doi:10.1002/hep.23280)

Haffner SM 2003 Pre-diabetes, insulin resistance, inflammation and CVD risk. Diabetes Research and Clinical Practice 61 S9-S18. (doi:10.1016/ S0168-8227(03)00122-0)

Hartstra AV, Bouter KEC, Backhed F \& Nieuwdorp M 2015 Insights into the role of the microbiome in obesity and type 2 diabetes. Diabetes Care 38 159-165. (doi:10.2337/dc14-0769)

Inzucchi SE, Bergenstal RM, Buse JB, Diamant M, Ferrannini E, Nauck M, Peters AL, Tsapas A, Wender R \& Matthews DR 2015 Management of hyperglycemia in type 2 diabetes, 2015: a patient-centered approach. Update to a position statement of the american diabetes association and the european association for the study of diabetes. Diabetes Care 38 140-149. (doi:10.2337/dc14-2441)

Jendle J, Nauck MA, Matthews DR, Frid A, Hermansen K, Düring M, Zdravkovic M, Strauss BJ \& Garber AJ 2009 Weight loss with liraglutide, a once-daily human glucagon-like peptide- 1 analogue for type 2 diabetes treatment as monotherapy or added to metformin, is primarily as a result of a reduction in fat tissue. Diabetes, Obesity and Metabolism 11 1163-1172. (doi:10.1111/j.14631326.2009.01158.x)

Jung J, Kim IY, Kim YN, Kim J, Shin JH, Jang Z, Lee H-S, Hwang G-S \& Seong JK 2012 1H NMR-based metabolite profiling of diet-induced obesity in a mouse mode. BMB Reports 45 419-424. (doi:10.5483/ BMBRep.2012.45.7.248)

Kopecký J, Hodný Z, Rossmeisl M, Syrový I \& Kozak LP 1996 Reduction of dietary obesity in aP2-Ucp transgenic mice: physiology and adipose tissue distribution. American Journal of Physiology $\mathbf{2 7 0}$ E768-E775.

Liu G, Yan T, Wang J, Huang Z, Chen X, Jia G, Wu C, Zhao H, Xue B, Xiao L, et al. 2013 Biological system responses to zearalenone mycotoxin exposure by integrated metabolomic studies. Journal of Agricultural and Food Chemistry 61 11212-11221. (doi:10.1021/ jf403401v)

Magnusson M, Wang TJ, Clish C, Engstrom G, Nilsson P, Gerszten RE \& Melander O 2015 Dimethylglycine deficiency and the development of diabetes. Diabetes 64 3010-3016. (doi:10.2337/ db14-1863)

Marso SP, Daniels GH, Brown-Frandsen K, Kristensen P, Mann JFE, Nauck MA, Nissen SE, Pocock S, Poulter NR, Ravn LS, et al. 2016 Liraglutide and cardiovascular outcomes in type 2 diabetes. New England Journal of Medicine 375 311-322. (doi:10.1056/ NEJMoa1603827)

Nicholson JK, Lindon JC \& Holmes E 1999 'Metabonomics': understanding the metabolic responses of living systems to pathophysiological stimuli via multivariate statistical analysis of biological NMR spectroscopic data. Xenobiotica 29 1181-1189. (doi:10.1080/004982599238047)

O'Rahilly S 1997 Science, medicine, and the future - non-insulin dependent diabetes mellitus: the gathering storm. British Medical Journal 314 955-959. (doi:10.1136/bmj.314.7085.955)

Pelantová H, Bugáňová M, Anýž J, Železná B, Maletínská L, Novák D Haluzík M \& Kuzma M 2015 Strategy for NMR metabolomic analysis of urine in mouse models of obesity-from sample collection to interpretation of acquired data. Journal of Pharmaceutical and Biomedical Analysis 115 225-235. (doi: 10.1016/j.jpba.2015.06.036)

Pelantová H, Bugáňová M, Holubová M, Šedivá B, Zemenová J, Sýkora D, Kaválková P, Haluzík M, Železná B, Maletínská L, et al. 2016a Urinary metabolomic profiling in mice with diet-induced obesity and type 2 diabetes mellitus after treatment with metformin, vildagliptin and their combination. Molecular and Cellular Endocrinology 431 88-100. (doi:10.1016/j.mce.2016.05.003)

Pelantová H, Bártová S, Anýž J, Holubová M, Železná B, Maletínská L, Novák D, Lacinová Z, Šulc M, Haluzík M, et al. 2016b Metabolomic profiling of urinary changes in mice with monosodium glutamateinduced obesity. Analytical and Bioanalytical Chemistry 408 567-578. (doi:10.1007/s00216-015-9133-0)

Salek RM, Maguire ML, Bentley E, Rubtsov D V, Hough T, Cheeseman M Nunez D, Sweatman BC, Haselden JN, Cox RD, et al. 2007 A metabolomic comparison of urinary changes in type 2 diabetes in mouse, rat, and human. Physiological Genomics 29 99-108. (doi:10.1152/physiolgenomics.00194.2006)

Schroeder JC, DiNatale BC, Murray IA, Flaveny CA, Liu Q, Laurenzana EM, Lin JM, Strom SC, Omiecinski CJ, Amin S, et al. 2010 The uremic toxin 3-indoxyl sulfate is a potent endogenous agonist for the human aryl hydrocarbon receptor. Biochemistry 49 393-400. (doi:10.1021/bi901786x)

Swann JR, Tuohy KM, Lindfors P, Brown DT, Gibson GR, Wilson ID, Sidaway J, Nicholson JK \& Holmes E 2011 Variation in antibioticinduced microbial recolonization impacts on the host metabolic phenotypes of rats. Journal of Proteome Research 10 3590-3603. (doi:10.1021/pr200243t)

van Can J, Sloth B, Jensen CB, Flint A, Blaak EE \& Saris WHM 2014 Effects of the once-daily GLP-1 analog liraglutide on gastric emptying, glycemic parameters, appetite and energy metabolism in obese, non-diabetic adults. International Journal of Obesity 38 784-793. (doi:10.1038/ijo.2013.162)

Waldram A, Holmes E, Wang Y, Rantalainen M, Wilson ID, Tuohy KM, McCartney AL, Gibson GR \& Nicholson JK 2009 Top-down systems biology modeling of host metabotype-microbiome associations in obese rodents. Journal of Proteome Research 8 2361-2375. (doi:10.1021/ pr8009885)

Waterfield C, Turton J, Scales M \& Timbrell J 1990 Taurine synthesis in isolated rat hepatocytes in suspension exposed to carbontetrachloride. Biochemical Society Transactions 18 1218-1219. (doi:10.1042/bst0181218) http://joe.endocrinology-journals.org

DOI: $10.1530 / J O E-16-0478$
(C) 2017 Society for Endocrinology Printed in Great Britain 
Waterfield CJ, Turton JA, Scales MDC \& Timbrell JA 1991 Taurine, a possible urinary marker of liver damage: a study of taurine excretion in carbon tetrachloride-treated rats. Archives of Toxicology 65 548-555. (doi:10.1007/BF01973715)

Wyss M \& Kaddurah-Daouk R 2000 Creatine and creatinine metabolism. Physiological Reviews 80 1107-1213.
Xia J, Psychogios N, Young N \& Wishart DS 2009 MetaboAnalyst: a web server for metabolomic data analysis and interpretation. Nucleic Acids Research 37 W652-W660. (doi:10.1093/nar/gkp356)

Xia J, Sinelnikov IV, Han B \& Wishart DS 2015 MetaboAnalyst 3.0-making metabolomics more meaningful. Nucleic Acids Research $4 \mathbf{3}$ W251-W257. (doi:10.1093/nar/gkv380)

Received in final form 3 January 2017

Accepted 30 January 2017

Accepted Preprint published online 30 January 2017
๑ 2017 Society for Endocrinology Printed in Great Britain
Published by Bioscientifica Ltd. 